\title{
Case Study of Aspiration during Swallowing in a Patient Following Traumatic Brain Injury
}

\author{
Mingwen Yu ${ }^{1}$, Wei Huang ${ }^{2}$, Jun Tang1, Ping Wan ${ }^{1 *}$ and Chunlei \\ Shan ${ }^{1 *}$ \\ ${ }^{1}$ School of Rehabilitation Medicine, Shanghai University of Traditional Chinese \\ Medicine, China \\ ${ }^{2}$ Department of Ear, Nose and Throat, No. 7 Hospital of Shanghai, China
}

\section{Case Report}

Volume 2 Issue 2

Received Date: October 25, 2017

Published Date: November 16, 2017

DOI: $10.23880 /$ ooaj-16000158

*Corresponding authors: Ping Wan, School of Rehabilitation Medicine, Shanghai University of Traditional Chinese Medicine, Shanghai, China; Tel: (86)13918610950; E-mail: 13918610950@163.com Chunlei Shan, School of Rehabilitation Medicine, Shanghai University of Traditional Chinese Medicine, Shanghai, China; Tel: (86)13816215058; E-mail: shanclhappy@163.com

\section{Abstract}

Object: To explore the cause of aspiration during swallowing in a patient with traumatic brain injury and examine various rehabilitation treatment mechanisms.

Materials and methods: A 47-year old male patient with traumatic brain injury, severe dysphagia, and nasal feeding demonstrated multiple causes of aspiration during swallowing by videofluoroscopic swallowing study (VFSS) and fiber optic endosopic evaluation of swallowing (FEES). Based on the results, a two-stage therapeutic strategy was employed: (1) cold balloon dilatation and vocal fold closure exercise; (2) the chin-down posture and vocal fold addition exercise. The oral transit time (OTT), pharyngeal transit time (PTT), initiation of laryngeal closure (ILC), and laryngeal closure duration (LCD) were noted. Data of both pre- and post- therapy from this case were analyzed by single subject A-B-C criterionalteration design.

Results: After the first stage of the therapy, compared with pre-therapy, OTT and PTT significantly increased $(\mathrm{p}<0.001)$, and ILC significantly decreased $(\mathrm{P}=0.004<0.05)$. After the second stage of the therapy using chin-down techniques, the LCD increased significantly $(\mathrm{P}<0.001)$, but the PTT also increased $(\mathrm{P}<0.001)$.

Conclusion: Aspiration in traumatic brain injury can result from complicated causes requiring an integrated strategy of treatments. This case study proves that the combination of cold balloon dilatation and chin-down swallowing can providerehabilitationand overcome swallowing disorder in traumatic brain injury.

Keywords: Traumatic Brain Injury; Aspiration during swallowing; Ice balloon dilatation; Chin-down; VFSS; FEES 


\section{Otolaryngology Open Access Journal}

Abbreviations: VFSS: Videofluoroscopic Swallowing Study; OTT: Oral Transit Time; PTT: Pharyngeal Transit Time; ILC: Initiation of Laryngeal Closure; LCD: Laryngeal Closure Duration; FEES: Fiberoptic Endoscopic Evaluation of Swallowing; CPD: Cricopharyngeal Dysfunction; DLE: Distance of Laryngeal Elevation; PA: Percent of Aspiration; CMA: Cricopharyngeal Muscle Achalasia; VR: Vallecular Residue; PSR: Pyriform Sinuses Residue

\section{Introduction}

Aspiration commonly occurs in patients with severe brain injury as a result of swallowing disorders. Mackay LE, et al. [1] noted that aspiration most commonly occurred during swallowing (77\%), before swallowing (41\%), as well as after swallowing (18\%). If the larynx does not close adequately during swallowing or in the presence of unilateral vocal fold immobility, the food/liquid bolus partly enters the airway partly during swallowing. Logemann JA [2] considered reduced laryngeal closure as the only etiology for aspiration during swallowing, while Steele CM, et al. [3] supported the measurement of tongue strength, anatomically normalized measures of hyoid movement, and bolus dwell time in the pharynx while the larynx remained open as parameters relevant to aspiration risk. But some authors did not differentiate between the aspiration before or during swallowing in their articles; neither by videofluoroscopic swallowing study (VFSS) nor by fiberoptic endoscopic evaluation of swallowing (FEES) examinations. Bingjie L [4] used quantitative VFSS analysis to confirm that pharyngeal transit time (PTT) was statistically associated with the prevalence of penetration-aspiration. The chin-down posture is the most frequently employed postural maneuver in the treatment of aspiration in neurogenic dysphagia caused by brain injury; this compensatory strategy is to redirect the flow of food and/or liquids to prevent aspiration and eliminate residue, thus keeping patients safe when eating and drinking, which is different from the rehabilitative strategies aiming to accelerate the recovery process. However, Terre R \& Mearin F [5] reported that only 50\% of the patients with acquired brain injury did not aspirate adapting the chin-down posture; however, $48 \%$ of the patients continued to aspirate in this posture as evidenced by VFSS. And Saconato M, et al. [6] found that the more severe the dysphagia, the less effective the chindown posture.

Causes of severe aspiration are diverse and complicated. So in the case of reduced laryngeal closure, an extended stay of the bolus in the pharynx will aggravate aspiration. So we suppose that it is less effective to treat aspiration, only in the chin-down posture, not solving the problem of extended PTT. Power ML [7] considered that deficits in oral sensation have longer delays in the onset of laryngeal ascent and prolong PTT after brain injury. Lee KL, et al. [8] proved that when dysphagic patients were given $5 \mathrm{ml}$ each of a sour tasting liquid, it enhanced the sensorimotor feedback in the oropharynx, thereby lowering the chances of penetrationaspiration caused by shortening of the oropharyngeal passage times. The authors further indicated that there existed a risk for the patients presenting with aspiration and thus safety should be considered as a priority for patients with severe aspiration while considering treatment strategies. Dou Z, et al. [9] compared two modes of balloon dilatation therapy (active and passive) on cricopharyngeal dysfunction (CPD) and speculated that sensory input from the inflated balloon affects the swallowing central pattern generator and motor response. Yabunaka K, et al. [10] made use of the upper esophageal balloon catheter dilatation to treat severe pharyngeal dysphagia (pharyngeal dysfunction and aspiration before, during, or after food swallowing), and demonstrated that pharyngeal dysphagia improved following dilatation of the esophageal inlet in $31 \%$ of patients. But the study did not elaborate the kind of aspiration (before, during, or after swallowing) and thus the exact treating mechanism of the upper esophageal balloon catheter dilatation cannot be analyzed. Through the diagnosis and treatment of a patient with serious aspiration secondary to traumatic brain injury, our case study potentially assumes that the cold balloon dilatation could enhance sensorimotor feedback in the oropharynx, and improve CPD by shortening the oral transit time (OTT) and PTT. Accordingly, efficiency of swallowing would be improved; and then combining with the chindown posture severe aspiration could be avoided and patients may be restored to an oral intake.

\section{Materials and methods}

\section{Basic Medical Information}

A 47-year old male patient was admitted on Oct 13th, 2015 at Shanghai Seventh People's Hospital with a history of fall against the side of his ship following which he lost consciousness. CT imaging of the head, chest, and abdomen revealed brain swelling, compressed brain stem peripheral cisterns, hiatal hernia of tentorium cerebelli, traumatic subarachnoid hemorrhage, basicranial fracture, the anterior and the lateral wall of the left maxillary sinus fracture, and left maxillofacial subcutaneous hemorrhage. 


\section{Otolaryngology Open Access Journal}

The patient was given all emergency treatment in the ICU. The patient was then transferred to the rehabilitation department on December 7th, 2015. He began to accept swallowing and speech therapy on December 21st, 2015.During the treatment the patient was sane with normal cognitive function, but could not maintain a normal sitting posture, but could control the head motion. His trachea cannula was removed, but nasal feeding was continued. He had a right-sided facial paralysis, but no aphasia. His phonation was severe, breathy, and harsh. FEES revealed a left paralyzed vocal cord fixed at the paramedian position on December 24th, 2015.

\section{Research Methods}

VFSS: VESS was carried out by an $800 \mathrm{~mA}$ digital subtraction angiography X-ray machine (Siemens, Germany). This system can collect real-time digital images $(7 / s)$ and can play repeated measurements of the various stages of swallowing time. Suspension of barium sulfate II type and thickener (Our diet swallow, Our diet, Guangzhou, China) was used as contrast. The patient was required to receive VFSS before, during, and after treatment. The patient was asked to swallow $5 \mathrm{ml}$ of the soft pureed food three times (swallowing every ten seconds), obtaining a total of nine videos and the following parameters were analyzed:

a) Distance of Laryngeal Elevation (DLE)-- The reference point of the laryngeal movement is the posteriorsuperior corner of the subglottic air column. The start of laryngeal elevation begins with the video frame exhibiting the first upward movement and ends with the video frame exhibiting the last downward movement. The normal value is $2 \mathrm{~cm}$ or more $[11,12]$.

b) Initiation of Laryngeal Closure (ILC)-- The bolus enters the pharynx (operationally defined as the posterior edge of the ramus of the mandible) and the first contact with the arytenoids and the epiglottis [13].

c) Laryngeal Closure Duration (LCD)-- The duration of the approximation of the arytenoid cartilages to the base of the epiglottis [13].

d) Oral Transit Time (OTT)-- Onset of the bolus movement in the mouth until the head of the bolus reaches the point where the lower rim of the mandible crosses the tongue base $[11,12]$.

e) Pharyngeal Transit Time (PTT)-- Arrival of the bolus head at the point where the lower rim of the mandible crosses the tongue base until the bolus tail passes through the cricopharyngeal region $[11,12]$.

f) Percent of Aspiration (PA)-- The percent of aspirated bolus to the total bolus arriving to the pharynx before swallowing [11,12]. Figure 1 represents the VFSS results of this patient before treatment

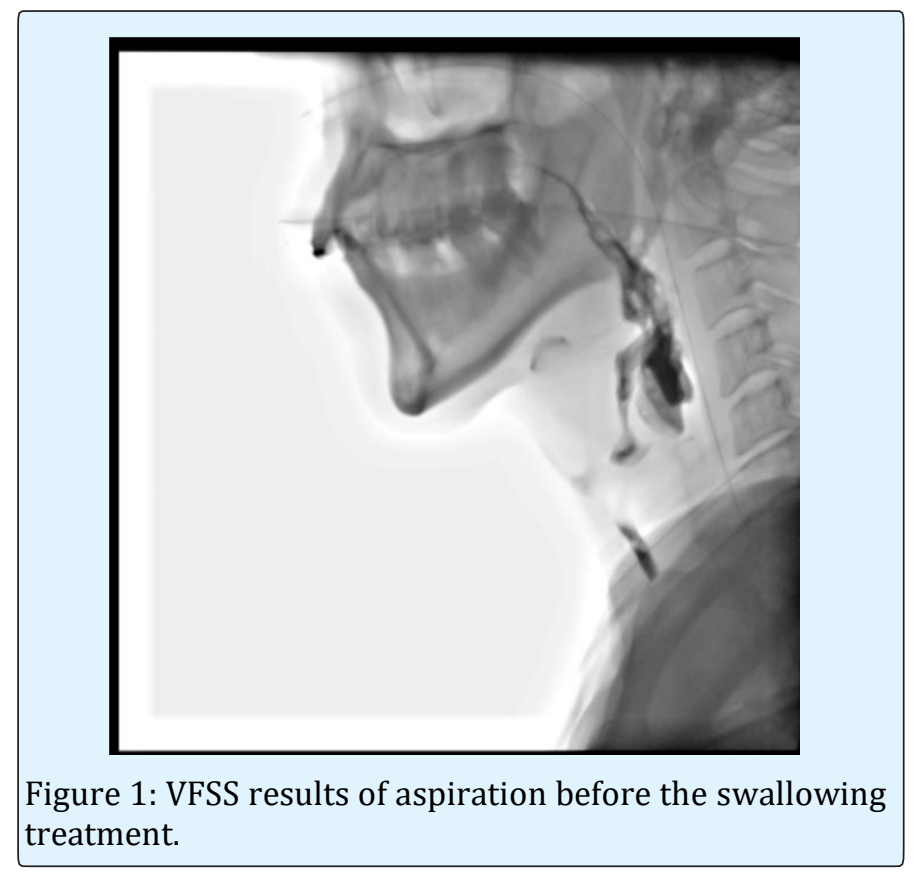

Intrarater/Inter-rate Agreement: One physician (P.W.) and two speech and language pathologists (M.W.Y and J.T), analyzed the digital file of the VFSS studies respectively, at three random times. A total of 9 videos of VFSS, with each of the parameters (DLE, ILC, LCD, OTT, PTT, and PA) had 81 evaluation values, and we randomly selected $30 \%$ of all measurements to calculate the intra-/ inter-rater reliability using SPSS 21.0 (IBM, USA). We calculated the intraclass correlation (ICC) to analyze the intra-/ inter-rater reliability. The inter-rater reliability as ICC calculation was 0.719 to 0.988 , and the intra-rater reliability as ICC calculation was 0.813 to 0.995 .

Swallowing treatment: The first VFSS displayed obvious aspiration during swallowing, OTT was $1.63 \mathrm{~s}$, and PTT was $10.33 \mathrm{~s}$, so we considered the patient's swallowing to be inefficient and his laryngeal closure to be reduced. As a security consideration, we adopted a two-stage treatment. In the first stage, indirect treatment strategies were used: cold balloon dilatation ( 2 weeks, 3 times per week, 8 times each time), along with laryngeal elevation exercisethe falsetto exercise, the Mendelsohn maneuver, and tongue base exercises were administered for 2 weeks. In the second stage, direct therapy was adapted, that is to swallow $5 \mathrm{ml}$ of the soft pureed food 10 times during the chin-down maneuver, and if the patient was choking, the trial would stop very quickly. In the meantime, the patient continued to do the falsetto exercise, the Mendelsohn 


\section{Otolaryngology Open Access Journal}

maneuver and the tongue base exercises, 3 times per week, for 1 week. All the treatment processes lasted for three weeks.

Statistical analysis: The data from this study was analyzed by a single baseline A-B-C criterion-alternation design, to observe the effect of treatment of aspiration during swallowing. All measurement variables of VFSS were collected, recorded, and analyzed using SPSS 21.0.

\section{Results}

We have classified and put in order the data of VFSS of this patient on December 29th, 2015 (before the treatment), January 12th, 2016(first stage treatment: stage 1), January 19th, 2016 (second stage treatment: stage 2) in Table 1. Tables 2-5 and Figures 1-4 show the significant change of the variables (OTT, PTT, ILC, and LCD), which were analyzed by a single subject research.

\begin{tabular}{|c|c|c|c|}
\hline VFSS (Lateral View) Radiographic & \multirow{2}{*}{$\begin{array}{c}\text { Before the treatment } \\
\text { Symptoms }\end{array}$} & Treatment & Treatment \\
\cline { 2 - 4 } & & Stage 1 & Stage 2 \\
\hline DLE(mm) & $16.56 \pm 2.58$ & $12.92 \pm 1.94$ & $14.77 \pm 2.22$ \\
\hline ILC(s) & $0.48 \pm 0.07$ & $0.24 \pm 0.32$ & $0.25 \pm 0$ \\
\hline LCD(s) & $0.49 \pm 0.15$ & $0.45 \pm 0.38$ & $0.67 \pm 0.11$ \\
\hline OTT (s) & $1.63 \pm 0.31$ & $1.03 \pm 0.20$ & $0.94 \pm 0.25$ \\
\hline PTT(s) & $10.33 \pm 2.08$ & $2.35 \pm 0.29$ & $3.17 \pm 2.20$ \\
\hline PDT(s) & 0 & 0 & 0 \\
\hline VR(\%) & $3 \% \pm 2 \%$ & $1 \% \pm 2 \%$ & $5 \% \pm 2 \%$ \\
\hline PSR(\%) & $5 \% \pm 0$ & $5 \% \pm 1 \%$ & Yes \\
\hline \multirow{2}{*}{ CMA } & Yes & Yes & $5 \mathrm{ml}$ swallow 2 times \\
\hline PA(\%) & $21 \% \pm 3 \%$ & $12 \% \pm 3 \%$ & 0 \\
\hline
\end{tabular}

Abbreviations: DLE, distance of laryngeal elevation; ILC, initiation of laryngeal closure; LCD, laryngeal closure duration; OTT, oral transit time; PTT, pharyngeal transit time; PDT, pharyngeal delay time; VR, vallecular residue; PSR, pyriform sinuses residue; CMA, cricopharyngeal muscle achalasia; PA, percent of aspiration.

Table 1: VFSS variables before treatment and during stage 1 and stage 2 of treatment.

Comparison of OTT Results before Treatment and at Stage 1 and Stage 2 of Treatment

\begin{tabular}{|c|c|c|c|}
\hline Measurements & Before the treatment & Stage 1 & Stage 2 \\
\hline 1 & 1.556 & 1.286 & 1 \\
\hline 2 & 1.792 & 1.143 & 0.571 \\
\hline 3 & 1.4 & 1.286 & 1.492 \\
\hline 4 & 1.57 & 1 & 0.571 \\
\hline 5 & 2.14 & 1 & 0.571 \\
\hline 6 & 2 & 1 & 0.857 \\
\hline 7 & 1.6 & 0.571 & 1 \\
\hline 8 & 1.5 & 1 & 1.429 \\
\hline 9 & 1.1 & 1 & $0.94 \pm 0.35$ \\
\hline $\mathrm{M} \pm \mathrm{SD}$ & $1.63 \pm 0.31$ & $1.03 \pm 0.21$ & -0.645 \\
\hline $\mathrm{Br}$ & 0.3543 & 0.3376 & 0.391 \\
\hline
\end{tabular}

Table 2: Data of OTT before treatment and at stage 1 and stage 2 of treatment. 


\section{Otolaryngology Open Access Journal}

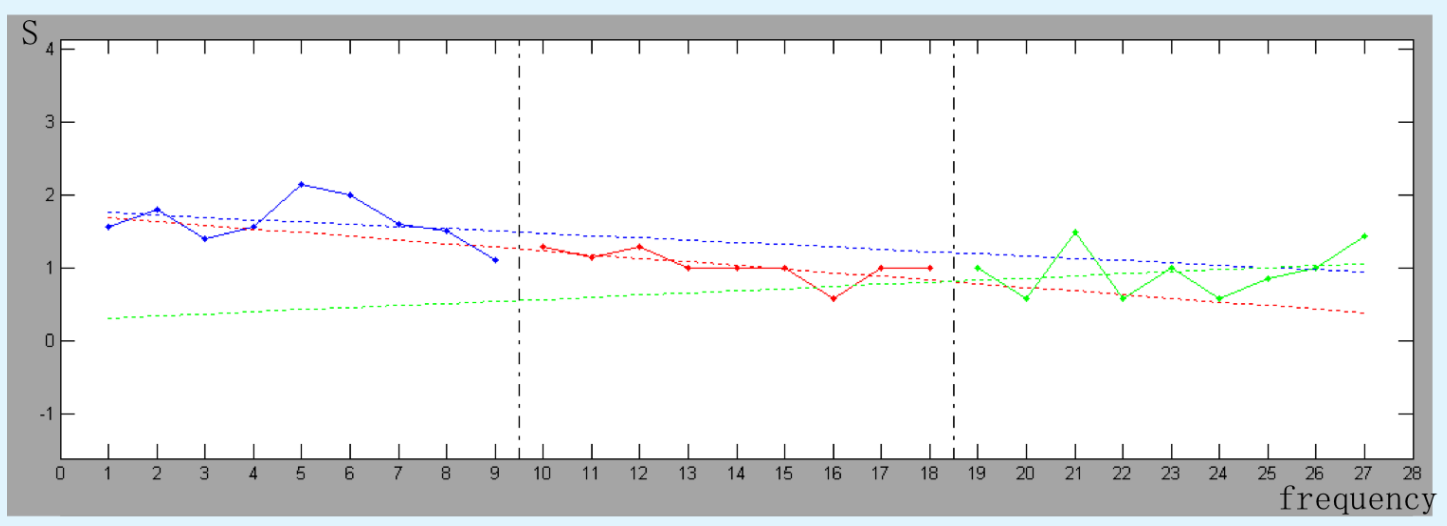

Figure 2: The changing trend of OTT before treatment and during stage 1 and stage 2 of treatment.

The data of OTT is summarized in table 2. There existed a significant change of OTT on the first stage treatment (p $<0.001)$. Figure 2 shows that after treatment of the first stage there was a significant decline in OTT, but there was no significance of OTT between the two stages $(p=0.391$ $>0.05)$.

\section{Comparison of PTT Results before Treatment and During Stage 1 and Stage 2 of Treatment}

\begin{tabular}{|c|c|c|c|}
\hline Measurements & Before the treatment & Treatment stage 1 & Treatment stage 2 \\
\hline 1 & 12.429 & 2.571 & 3.285 \\
\hline 2 & 10 & 2 & 3 \\
\hline 3 & 5.7 & 2.285 & 3 \\
\hline 4 & 12.571 & 2.714 & 3.571 \\
\hline 5 & 10.14 & 2 & 3 \\
\hline 6 & 10 & 2.285 & 3.714 \\
\hline 7 & 12.143 & 2.714 & 3 \\
\hline 8 & 10 & 2.571 & 3 \\
\hline $\mathrm{M} \pm \mathrm{SD}$ & 10 & 2 & $3.17 \pm 2.20$ \\
\hline $\mathrm{Br}$ & $10.33 \pm 2.08$ & $2.35 \pm 0.29$ & -0.5937 \\
\hline $\mathrm{P}$ & -0.4761 & -0.413 & 0 \\
\hline
\end{tabular}

Table 3: PTT data before treatment and during stage 1 and stage 2 of treatment.

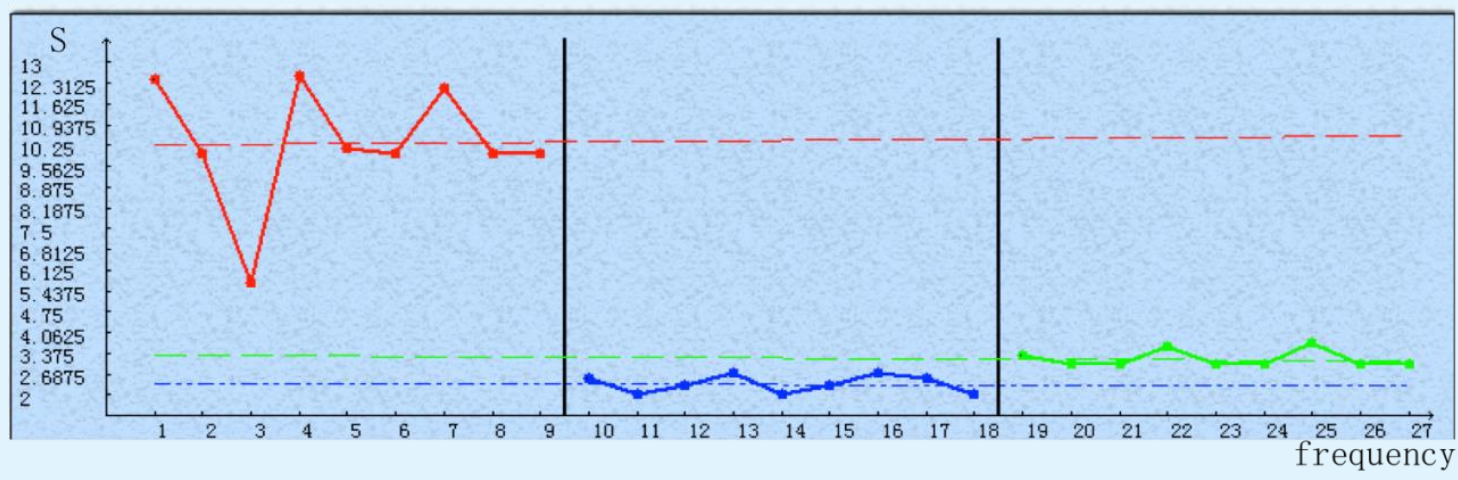

Figure 3: The trend graph of PTT before treatment and during stage 1 and stage 2 of treatment. 


\section{Otolaryngology Open Access Journal}

There was a significant change of PTT data after the first stage treatment $(\mathrm{p}=0<0.001)$.Figure 3 shows that after the treatment of the first stage, there was a significant decline in PTT. However, there was a significant rebound after the treatment of stage $2(\mathrm{p}=0<$ 0.001 ), probably reflected that the patient prolonged the swallowing motion as a compensation for an effort to reduce aspiration.

\section{Comparison of ILC Results before Treatment and During Stage 1 and Stage 2 of Treatment}

\begin{tabular}{|c|c|c|c|}
\hline Measurements & Before the treatment & Stage 1 & Stage 2 \\
\hline 1 & 0.429 & 0.143 & 0.143 \\
\hline 2 & 0.57 & 0.143 & 0.286 \\
\hline 3 & 0.43 & 0.286 & 0.143 \\
\hline 4 & 0.429 & 0.143 & 0.286 \\
\hline 5 & 0.571 & 0.571 & 0.286 \\
\hline 6 & 0.43 & 0.143 & 0.286 \\
\hline 7 & 0.429 & 0.143 & 0.428 \\
\hline 8 & 0.571 & 0.286 & 0.143 \\
\hline 9 & 0.43 & 0.286 & 0.286 \\
\hline $\mathrm{M} \pm \mathrm{SD}$ & $0.48 \pm 0.07$ & $0.24 \pm 0.32$ & $0.25 \pm 0$ \\
\hline $\mathrm{Br}$ & -0.8333 & -0.5212 & -0.5372 \\
\hline $\mathrm{P}$ & 0.004 & & 0.677 \\
\hline
\end{tabular}

Table 4: ILC data before, stage 1 and stage 2 of treatment.

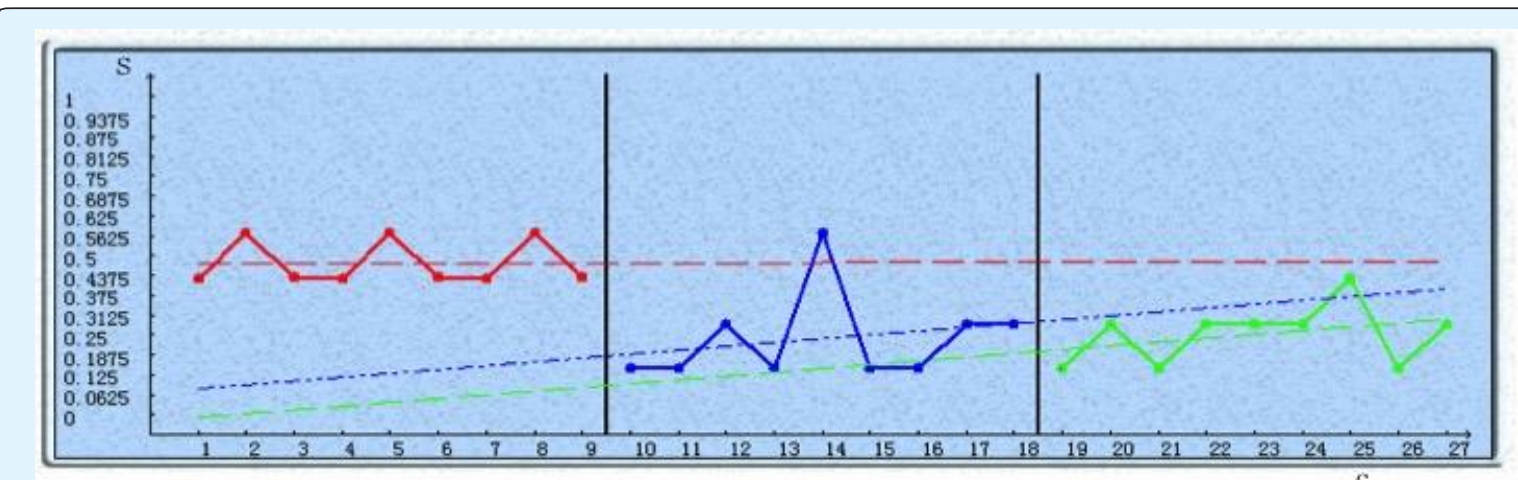

frequency

Figure 4: The ILC trend graph before treatment and during stage 1 and stage 2 of treatment.

The data of ILC is summarized in table 4. There existed a significant change of ILC on the first stage treatment $(\mathrm{p}=0.004<0.05)$. Figure 4 shows that after treatment of the first stage there was a significant decline in ILC, but there was no significance of ILC between the two stages $(p=0.677>0.05)$.

\section{Comparison of LCD Results before Treatment and During Stage 1 and Stage 2 of Treatment}

\begin{tabular}{|c|c|c|c|}
\hline Measurements & Before the treatment & Stage 1 & Stage 2 \\
\hline 1 & 0.429 & 0.429 & 0.571 \\
\hline 2 & 0.429 & 0.429 & 0.714 \\
\hline 3 & 0.571 & 0.571 & 0.714 \\
\hline 4 & 0.571 & 0.429 & 0.571 \\
\hline 5 & 0.429 & 0.429 & 0.714 \\
\hline 6 & 0.571 & 0.571 & 0.571 \\
\hline 7 & 0.429 & 0.429 & 0.714 \\
\hline
\end{tabular}




\section{Otolaryngology Open Access Journal}

\begin{tabular}{|c|c|c|c|}
\hline 8 & 0.571 & 0.286 & 0.714 \\
\hline 9 & 0.429 & 0.429 & 0.714 \\
\hline $\mathrm{M} \pm \mathrm{SD}$ & $0.49 \pm 0.15$ & $0.45 \pm 0.38$ & $0.67 \pm 0.11$ \\
\hline $\mathrm{Br}$ & -0.6583 & -0.0629 & -0.5833 \\
\hline $\mathrm{P}$ & \multicolumn{2}{|c|}{0.2285} & 0 \\
\hline
\end{tabular}

Table 5: LCD data before treatment and during stage 1 and stage 2 of treatment.

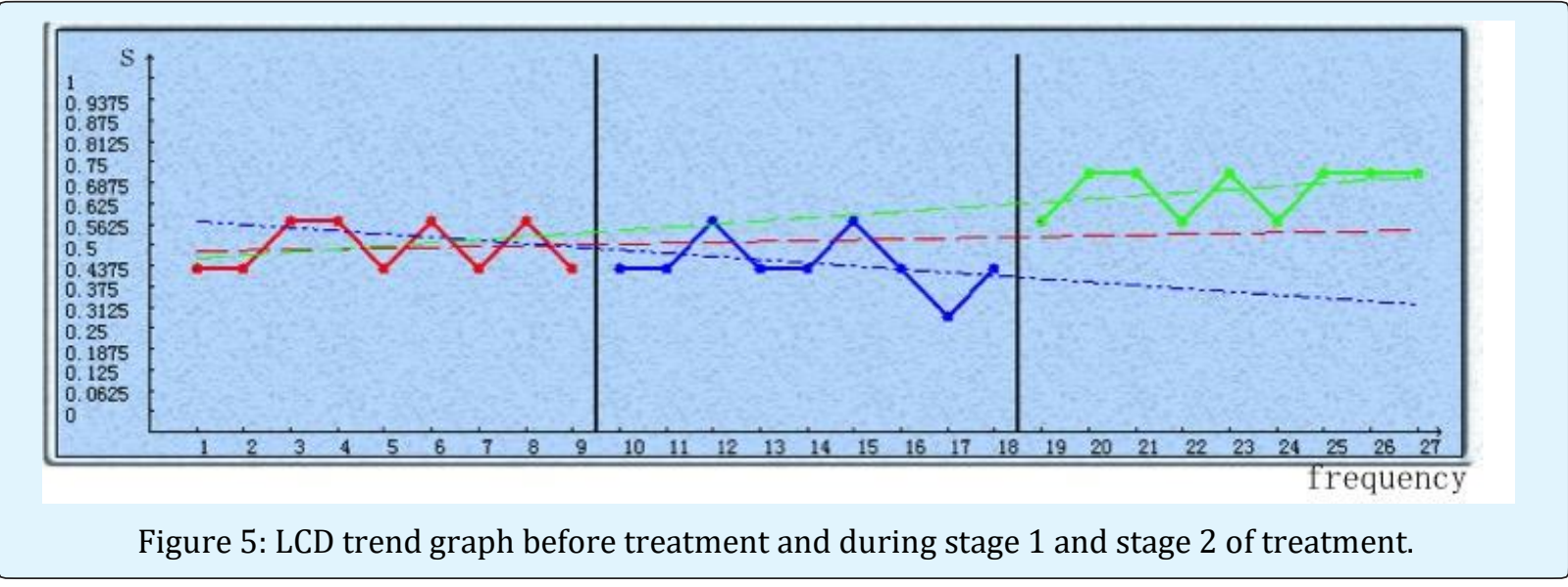

The data of LCD is summarized in table 5. There existed a significant change of LCD on the second stage treatment $(p=0.004<0.05)$. Figure 5 shows that after the second stage of treatment with the addition of chin-down swallowing, there was a significant increase in LCD.

\section{Discussion}

The patient had been receiving nasal feeding during his in-patient stay in the ICU for two months, and then he was transferred to the department of rehabilitation. On December 29th, 2015, he accepted VFSS, and was detected with silent aspiration, mainly during swallowing; the average percent of aspiration was $21 \%$. In addition, the patient had left vocal cord paralysis which led to reduced laryngeal closure during swallowing, which further pointed to aspiration during swallowing. In addition, VFSS for OTT and PTT was far beyond the normal range. The patient needed a full swallow divided into five small actions, so $95 \%$ of the bolus over-stayed in the pharynx could enter the esophagus at last. The brain CT had showed brain stem peripheral cisterns compressed (hiatal hernia of tentorium cerebelli), considered as lower motor neuron injury, comprehensively excluding the possibility of cricopharyngeal muscle achalasia (CMA) [14], but the base of the tongue and pharyngeal constrictor muscle contraction forces are weakened, and larynx elevation is not timely. However, in this study, the patient's distance of laryngeal elevation was in the normal range, the patient was left with CPD.

FEES on the December 24th, 2015 detected the patient with bilateral pyriform sinuses to have accumulated a lot of saliva, and when using the tip of mirror to touch the epiglottis, no cough or swallowing reflex was observed, which proved the patient with deficits in pharyngeal sensation. Thus, we surmise that although by triggering the pharyngeal swallowing in normal time (PDT $=0$ ), but the deficits in pharyngeal sensation made it impossible to form effective swallowing, distance of laryngeal elevation became inadequate (although the patient had normal distance of laryngeal elevation), the tongue base posterior movement and pharyngeal constrictors muscle contraction reduced, leading to the patient's CPD, and the extended OTT, and PTT.

Our results are consistent with the Terré [15] study that found most of the traumatic brain injury patients suffer from dysphagia. Most frequent early findings include an increase in OTT and alterations in lingual control, aspirations are quite frequent, and more than half are silent. The results are partially similar to Jang YY, et al. [16] report where central and peripheral vocal cord paralyses are compared in persons who complain of swallowing difficulties. They found that delay in triggering and poor coordination of swallowing (PTT and PDT prolonged) were profound in patients with central vocal cord paralysis, but dysfunction in peripheral vocal 


\section{Otolaryngology Open Access Journal}

cord paralysis may originate from poor pharyngeal movement.

In order to recover the patient's ability for oral intake, the treatment plan was carried out in two phases. In the first stage, continuous nasogastric nutrition was continued and use of indirect therapy included the ice balloon dilatation therapy, combined with tongue base exercises, Mendelsohn maneuver, and laryngeal elevation exercise-the falsetto exercise $[17,18]$. On January 12th, 2016 VFSS showed the patient's OTT was about one second, and vallecula had no residue, indicating the patient's tongue muscle had returned to a normal strength, thus the strength needed to push down the bolus increased. After the first stage of treatment, the VFSS found that there was a significant decline in ILC $(p=0.004<0.05)$, indicating that deficits in swallowing sensation has been significantly enhanced, and the tongue base and pharyngeal constrictor muscle contraction power increased, the PTT shortened. Though still $12 \%$ of aspiration was present during swallowing, but compared with before treatment the aspiration had decreased significantly compared to before treatment aspiration of $21 \%$, which is in accordance with the opinion of Yabunaka $\mathrm{K}$, et al. [10] who found that after videofluoroscopyguided balloon dilatation, aspiration was decreased. This phase focused on the use of ice balloon dilatation therapy while we used the Mendelsohn maneuver and the laryngeal elevation exercise-the falsetto exercise, the purpose was to increase the laryngeal closure. From the actual effect of treatment, the patient's OTT, PTT, tongue and pharyngeal constrictor muscle contraction improved significantly, indicating that the patient's swallowing synergy had been significantly improved. However since the left vocal cord paralysis still persisted, despite the use of many methods to promote laryngeal closure, no significant improvement in LCD was observed and $12 \%$ of aspiration still persisted.

Through the first phase of treatment, the patient's swallowing function improved, so we increased the direct therapy in the second stage. While promoting training glottal closure, combined with the use of the chin-down swallowing, resulted in the patient eating mushy food without choking. After direct therapy three times a week, the patient accepted to undergo VFSS again on January 19th, 2016, the whole inspection process was used in the chin-down position. Aspiration was reduced from $12 \%$ to 0 , LCD before and after treatment of this stage showed statistical significance $(\mathrm{p}<0.001)$, which explained that the chin-down position increases the laryngeal closure duration, so as to effectively prevent swallowing of aspiration.

Although the left vocal cord paralysis of this patient do not improve, with the chin-down posture, the anterior pharyngeal wall is pushed posteriorly and the tongue base and epiglottis are pushed closer to the posterior pharyngeal wall. This puts the epiglottis in a more protective position; So the chin-down posture could narrow the laryngeal entrance, and increase the time and strength of the vocal fold closure by applying extrinsic pressure, meanwhile widen the valleculae to prevent the bolus entering the airway. Thus, the chin-down posture is helpful if the patients who have reduced tongue base retraction and reduced airway entrance closure [19,20]. But we found that after the use of the chin-down posture, the patient's PTT appeared to be more significantly prolonged $(\mathrm{P}<0.001)$, we speculate that is because the part of bolus aspirated before would enter the oesophagus again, which spent more time than before. Due to prolonged PTT, some of swallowing problems still persist. But we consider that the patient is still young (47 years old), which means he has more recovering talent after traumatic brain injury. We believe he will swallow more and more normally following increased oral feeding activities later, and then we could be informed about his swallow ability by phoning him.

\section{Conclusion}

VFSS and FEES are gold standards to evaluate dysphagia. Once the causes for swallowing disorders are identified, a comprehensive and systematic treatment plan can relieve dysphagia. The ice balloon dilatation can also be a good sensory stimulation technology, which benefits patients with CPD of deficits in pharyngeal sensation.LCD increases during chin-down swallowing, effectively reducing aspiration.

\section{Limitation and Outlook}

The study was analyzed by experimental design (the total percent of aspiration - the half percent of aspiration no aspiration), to observe the impact of aspiration during swallowing. The advantage of single baseline A-B-C design is to precisely observe such aspiration and how to get the gradual improvement, hence the design method is more appropriate than the A-B-A-B design [21].

The reasons of aspiration in this case were more complex, so we used a step-wise approach to reach treatment goals (eliminate aspiration).The first stage 


\section{Otolaryngology Open Access Journal}

focused on the use of ice balloon dilatation therapy and the second phase focused on the use of chin-down swallowing. Although this study focused on observation of the ring pharyngeal muscle ice balloon dilation effect, it is difficult to explain the ice balloon dilatation therapy is the only way to the first stage of the avoid aspiration.

Fortunately, the second phase using the chin-down position to improve mild to moderate aspiration effect was significant, and with high reliability and high degree.

This case study can be used as a preliminary test to verify the sensory stimulation effect of ice balloon dilatation, which benefits patients with aspiration. Future still needs to further expand the sample size to verify cricopharyngeal ice balloon dilatation in improving swallowing.

Funding: This study was funded by Key Specialty Construction Project of Pudong Health and Family Planning Commission of Shanghai (Grant No. PWZz201301).

\section{References}

1. Mackay LE, Morgan AS, Bernstein BA (1999) Swallowing Disorders in Severe Brain Injury: Risk Factors Affecting Return to Oral Intake. Arch Phys Med Rehabil 80(4): 365-371.

2. Logemann JA (1998) Evaluation and Treatment of Swallowing Disorders $2^{\text {nd }}(\mathrm{edn})$, Pro-Ed, Texas, USA, pp: 92-107.

3. Steele CM, Cichero JA (2014) Physiological factors related to aspiration risk: a systematic review. Dysphagia 29(3): 295-304.

4. Bingjie L, Tong Z, Xinting S, Jianmin X, Guijun J (2010) Quantitative videofluoroscopic analysis of penetration-aspiration in post-stroke patients. Neurol India 58(1): 42-47.

5. Terré R, Mearin F (2012) Effectiveness of chin-down posture to prevent tracheal aspiration in dysphagia secondary to acquired brain injury. A videofluoroscopy study. Neurogastroenterol Motil. 24(5): 414-419.

6. Saconato M, Chiari BM, Lederman HM, Gonçalves M (2016) Effectiveness of Chin-tuck Maneuver to
Facilitate Swallowing in Neurologic Dysphagia. Int Arch Otorhinolaryngol 20(1): 13-17.

7. Power ML, Hamdy S, Singh S, Tyrrell PJ, Turnbull I, et al. (2007) Deglutitive laryngeal closure in stroke patients. J Neurol Neurosurg Psychiatry 78(2): 141146.

8. Lee KL, Kim DY, Kim WH, Kim EJ, Lee WS, et al. (2012) The influence of sour taste on Dysphagia in brain injury: blind study. Ann Rehabil Med 36(3): 365-370.

9. Dou Z, Zu Y, Wen H, Wan G, Jiang L, et al. (2012) The effect of different catheter balloon dilatation modes on cricopharyngeal dysfunction in patients with dysphagia. Dysphagia 27(4): 514-520.

10. Yabunaka K, Konishi H, Nakagami G,Matsuo J, Noguchi A, Sanada H (2015) Videofluoroscopy-guided balloon dilatation for treatment of severe pharyngeal dysphagia. Diagn Interv Radiol 21(2): 173-176.

11. Logemann JA, Pauloski BR, Rademaker AW, Kahrilas PJ (2002) Oropharyngeal swallow in younger and older women: videofluoroscopic analysis. J Speech Lang Hear Res JSLHR 45(3): 434-445.

12. Ping Wan, Oliver Ding, Lequn Zhu (2011) Application of videofluoroscopic Swallowing Study (VFSS) to oralpharyngeal dysphagia. Chinese Rehabilitaion Theory and Practice 17(12): 1107-1111.

13. Park T, Kim Y, Ko DH, McCullough G (2010) Initiation and Duration of Laryngeal Closure During the Pharyngeal Swallow in Post-Stroke Patients. Dysphagia 25(3): 177-182.

14. Wan P, Chen X, Zhu L, Xu S, Huang L, et al. (2016) Dysphagia Post Subcortical and Supratentorial Stroke. J Stroke Cerebrovasc Dis 25(1): 74-82.

15. Terré R, Mearin F (2007) Videofluoroscopy quantification of laryngotracheal aspiration outcome in traumatic brain injury-related oropharyngeal dysphagia.Rev Esp Enferm Dig 99(1): 7-12.

16. Jang YY, Lee SJ, Jeon JY, Lee SJ (2012) Analysis of video fluoroscopic swallowing study in patients with vocal cord paralysis. Dysphagia 27(2): 185-190.

17. McCullough GH, Kim Y (2013) Effects of the Mendelsohn maneuver on extent of hyoid movement and UES opening post-stroke. Dysphagia 28(4): 511519. 


\section{Otolaryngology Open Access Journal}

18. Sze WP, Yoon WL, Escoffier N, Rickard Liow SJ (2016) Evaluating the Training Effects of Two Swallowing Rehabilitation Therapies Using Surface Electromyography-Chin Tuck Against Resistance (CTAR) Exercise and the Shaker Exercise. Dysphagia 31(2): 1-11.

19. Welch MV, Logemann JA, Rademaker AW, Kahrilas PJ (1993) Changes in pharyngeal dimensions effected by chin tuck. Arch Phys Med Rehabil 74(2): 178-181.
20. Shanahan TK, Logemann JA, Rademaker AW, Pauloski BR, Kahrilas PJ (1993) Chin-down posture effect on aspiration in dysphagic patients. Arch Phys Med Rehabil 74(7): 736-739.

21. Kazdin Alan E (2010) Single-Case research designs: methods for clinical and applied settings.2nd ed. Oxford University Press, London, England, pp: 189191. 